Национальный медицинский исследовательский центр эндокринологии, Москва, Россия

В статье представлен обзор научных работ, посвященных проблеме репродуктивного здоровья мужчин с синдромом Клайнфельтера (СК) с позиции патогенеза, возможности обеспечения биологического отцовства и риска возникновения хромосомных аномалий у потомства. Освещены известные на сегодняшний день обстоятельства развития патологического состояния гонад с внутриутробного периода развития.

Несмотря на то что СК является одной из наиболее частых причин мужского бесплодия, ассоциированного с хромосомными аномалиями, ввиду вариабельности клинических проявлений данного заболевания до широкого внедрения неинвазивного пренатального тестирования доля пациентов, выявленных до наступления пубертата, не превышала 10\%. Согласно приведенным в статье результатам исследований, репродуктивный потенциал у мужчин с СК зачастую снижен уже в период раннего детства, что целесообразно учитывать при выборе дальнейшей тактики ведения пациентов.

В литературе имеются данные о единичных случаях наступления спонтанной беременности от мужчин с СК, в связи с чем обеспечение биологического отцовства у данной группы пациентов зачастую возможно лишь путем применения хирургических методов экстракции сперматозоидов и вспомогательных репродуктивных технологий. В статье подробно рассмотрены такие методы, как хирургическая экстракция сперматозоидов (testicular sperm extraction, TESE) и микрохирургическая экстракция сперматозоидов (microdissection testicular sperm extraction, mTESE) с позиции их эффективности и безопасности для пациента, а также представлены факторы, потенциально способные повлиять на исход операции. Оптимальным периодом проведения названных манипуляций является возраст пациента от 18 до 30 лет, а применение перечисленных хирургических методов у подростков вызывает бурные дискуссии.

Проанализированные в статье результаты исследований позволяют предположить, что риск передачи хромосомных аномалий потомству является, скорее, низким, что, однако, не исключает необходимости проведения медико-генетического консультирования, а также предимплантационной или внутриутробной диагностики.

КЛЮЧЕВЫЕ СЛОВА: синдром Клайнфельтера; мужское бесплодие; вспомогательные репродуктивные технологии; экстракция сперматозоидов из яичка TESE; микрохирургическая экстракция сперматозоидов яичка $\mathrm{mTESE}$.

\title{
KLINEFELTER SYNDROME: LITERATURE REVIEW ON USING MODERN METHODS OF ASSISTED REPRODUCTIVE TECHNOLOGIES
}

\author{
(c) Maria V. Vorontsova*, Natalia Yu. Kalinchenko
}

Endocrinology Research Centre, Moscow, Russia

The article reviews scientific papers devoted to the problem of reproductive health in men with Klinefelter syndrome (KS). Pathogenesis from a very early age (in utero), the possibility of ensuring biological paternity upon reaching sexual maturity and the risk of chromosomal abnormalities in offspring are discussed. Despite the fact that KS is one of the most common causes of male infertility associated with chromosomal abnormalities, due to the variability of clinical manifestations the proportion of patients identified before puberty did not exceed $10 \%$ before the widespread introduction of non-invasive prenatal testing. According to the research results presented in the article, the reproductive potential of males with KS is often already reduced in early childhood. These circumstances should be considered when choosing further patient management tactics.

There are few reports on the onset of spontaneous pregnancy in the case of KS, so ensuring biological paternity in this group of patients is often possible only using surgical methods of sperm extraction and assisted reproductive technologies. This article discusses methods like testicular sperm extraction (TESE) and microdissection testicular sperm extraction (mTESE) in terms of their effectiveness and safety for the patient, and the factors influencing the outcome of the operation. The optimal period of these manipulations seems to be the patient's age from 18 to 30 years, although the feasibility of adolescent boys undergoing the aforementioned procedures is highly controversial.

The research papers presented in the article suggest that the risk of transmitting chromosomal abnormalities to offspring is rather low, which does not exclude the need for medical and genetic counseling to explain all possible risks to the patient. Preimplantation or intrauterine diagnostics are also deemed necessary.

KEYWORDS: Klinefelter syndrome; male infertility; assisted reproductive technologies; testicular sperm extraction (TESE); microsurgical testicular sperm extraction (ITTESE). 


\section{ОПРЕДЕЛЕНИЕ СИНДРОМА, КЛИНИЧЕСКАЯ КАРТИНА}

Мужское бесплодие (МБ) является одной из актуальных проблем современного общества. Среди ведущих причин МБ выделяют болезни, ассоциированные с хромосомными аномалиями, из которых наиболее часто встречается синдром Клайнфельтера (СК) [1]. По данным различных популяционных исследований, доля пациентов с СК среди мужчин, страдающих бесплодием, составляет от 2,7 до $11 \%[2,3]$.

СК впервые описан в 1942 г. врачом Гарри Клайнфельтером как заболевание, обусловленное нарушением секреции андрогенов и повышенной секрецией фолликулостимулирующего гормона, проявляющееся гинекомастией и азооспермией. В основе синдрома лежит присутствие одной или более дополнительных $\mathrm{X}$ хромосом у мужчины. Кариотип 47XXY встречается наиболее часто, анеуплоидия 48XXXY или мозаичная форма 47XXY/46XY составляют около $20 \%$ случаев, тогда как другие варианты хромосомных числовых аномалий встречаются очень редко [4].

Предполагаемая частота СК составляет примерно 155 на 100 тыс. мальчиков, рожденных живыми (примерно 1:660) [5]. При этом только у 25\% больных мальчиков и мужчин заболевание выявляется, и менее чем в $10 \%$ случаев диагноз выставляется до начала пубертата [6]. Низкая выявляемость обусловлена значительной вариабельностью клинических проявлений при СК. Однако в последнее время в связи с повсеместным внедрением неинвазивного пренатального тестирования пренатальная и ранняя диагностика СК неуклонно растет. Так, в 2015 г. в Австралии были опубликованы данные, что $21 \%$ всех диагнозов СК были определены в пренатальном периоде и только в 16\% - в пубертатном [7], что обуславливает актуальность определения возможности раннего лечения и сохранения фертильности у данной группы пациентов.

Классическое описание СК Гарри Клайнфельтером включало ряд облигатных признаков: малый объем тестикул (до 4 мл), гипогонадотропный гипогонадизм, азооспермию, евнухоидное строение тела, гинекомастию, когнитивные отклонения вплоть до умственной отсталости [8].

На сегодняшний день представление о клинической картине СК значительно расширилось. Установлено, что проявления вариабельны и могут включать такие признаки, как позднее или неполное половое созревание, склонность к полноте и развитию метаболического синдрома, остеопороза и сердечных заболеваний в зрелом возрасте [4, 9], а также ряд психологических и психических особенностей, кроме умственной отсталости, - сложности в обучении и развитии речи, иногда склонность к агрессивному поведению [10]. В связи с разнообразием и зачастую стертостью клинических проявлений большинство пациентов выявляются случайно при обследовании по поводу бесплодия.

Для взрослых пациентов с СК характерны склонность к коморбидности и снижение продолжительности жизни. К распространенным компонентам коморбидного статуса относятся: варикозное расширение вен, тромбозы, эмболии, сахарный диабет 2-го типа, частые переломы, эпилепсия и другие [9]. По данным исследования
Bojesen et al., в котором рассматривались когорты пациентов из Дании и Великобритании [11], риск летального исхода коморбидных состояний повышен на $50 \%$.

\section{БЕСПЛОДИЕ ПРИ СИНДРОМЕ КЛАЙНФЕЛЬТЕРА}

Фетальный и младенческий периоды, мини-пубертат

Одним из классических признаков СК является малый объем тестикул, обусловленный атрофией и гиалинизацией семенных канальцев. По данным исследований абортивного материала, количество герминативных клеток снижено уже в период внутриутробного развития (18-22 нед гестации) [12], в то время как число, плотность семенных канальцев и мезенхимальные структуры соответствуют норме. Отмечается и снижение дифференцировки гоноцитов в пресперматогонии. До настоящего времени точно не установлены механизм гиалинизации семенных канальцев и период его начала. Так, Winge и коллеги с помощью РНК-секвенирования показали, что уже в фетальный период отмечается повышенная экспрессия ряда генов X-хромосомы, приводящих к гиалинозу семенных канальцев [13]. Вместе с тем Van Saen и соавт. выявили отсутствие фиброза тестикулярной ткани в период эмбрионального развития (срок 19-21 нед) при сопоставимом с контрольными образцами количестве герминативных клеток [14].

В норме в возрасте до 6 мес у детей отмечается период мини-пубертата, характеризующийся повышением уровней гонадотропинов и половых стероидов [15]. Имеющиеся на сегодняшний день результаты исследований о течении мини-пубертата у пациентов с СК противоречивы. Так, в исследовании Lahlou и коллег было показано [16], что у мальчиков с СК концентрация тестостерона (Т) крови повышается в 1-й месяц жизни, однако далее снижается ниже среднего уровня популяции здоровых мальчиков, что указывает на функциональные нарушения клеток Лейдига. В то же время уровни лютеинизирующего гормона (ЛГ), фолликулостимулирующего гормона (ФСГ), ингибина Б (ИнгБ) и антимюллерова гормона (АМГ) остаются в норме. Напротив, в исследовании Aksglaede et al. продемонстрирован высокий уровень всех гормональных показателей (общий Т, свободный Т, ЛГ, ФСГ, а также соотношение ФСГ/ИнгБ), кроме ИнгБ и глобулина, связывающего половые стероиды, которые были в норме [17].

Таким образом, есть данные о том, что репродуктивный потенциал мальчиков с СК снижен уже на ранних стадиях развития, что позволяет рассматривать период мини-пубертата как время для исследований гипофизарно-гонадной оси и функциональных свойств тестикулярной ткани у пациентов с СК, а также как возможность ранней диагностики и терапии.

\section{Детство и пубертат}

Значимые изменения в гормональном профиле у пациентов с СК развиваются с началом пубертата. У большинства мальчиков с СК пубертат начинается спонтанно - объем тестикул увеличивается в среднем до 6 мл за счет пролиферации клеток Сертоли и интерстиция, повышается концентрация Т крови [18]. Далее с повышением Т связывают резкое начало и/или ускорение уже имеющейся дегенерации и гиалинизации семенных канальцев, ускорение деструкции герминативных клеток, 
сокращение численности клеток Сертоли, что приводит к уменьшению объема тестикул до препубертатных значений 2-4 мл [14, 15]. В гормональном профиле при классическом варианте СК наблюдается резкое повышение ФСГ. Нередко повышается и ЛГ в ответ на снижающийся уровень Т. Секреция ИнгБ, концентрация которого высока непосредственно перед началом полового созревания, после запуска пубертата резко снижается. Учитывая, что секреция ИнгБ зависит от функции герминативных клеток, а клетками Сертоли регулируется в меньшей степени, снижение уровня гормона следует рассматривать, скорее, как показатель функции герминативных клеток.

Таким образом, к концу пубертата у мальчиков с СК отмечается прогрессирующая деструкция семенных канальцев, что ведет к сокращению объема тестикул до 2-4 мл. Также имеется относительный гипергонадотропный гипогонадизм (высокие уровни ФСГ, ЛГ, Т на нижней границе нормы, низкий уровень ИнгБ), что указывает на функциональную несостоятельность клеток Лейдига и клеток Сертоли, а также на деструкцию герминативных клеток.

\section{Фертильность пациентов с СК}

В связи с гиалинозом семенных канальцев и дегенерацией герминативных клеток для СК характерна азооспермия. При этом результаты исследований свидетельствуют о том, что в сперматогенной ткани остаются островки [14, 18], теоретически способные продуцировать жизнеспособные сперматозоиды. В литературе встречаются единичные описания спонтанной беременности от мужчин с СК. Тем не менее в подавляющем большинстве случаев единственной возможностью отцовства у мужчин с СК является применение хирургических методов экстракции сперматозоидов и вспомогательных репродуктивных технологий.

Учитывая патогенез заболевания, его прогрессирующее течение, необходимость инициации заместительной гормональной терапии во многих случаях, возникает ряд вопросов относительно выбора тактики ведения пациентов с СК для обеспечения возможности биологического отцовства. Какие из имеющихся методов хирургической экстракции спермы наиболее эффективны? Какова частота положительных результатов операций и есть ли предикторные факторы успешности вмешательства? Каков наиболее благоприятный возраст проведения экстракции спермы и как влияет криопрезервация на качество сперматозоидов? Какой период начала заместительной гормональной терапии оптимален и какой эффект она оказывает на результаты экстракции? Насколько высок риск возникновения у потомства хромосомных нарушений в половых хромосомах и в аутосомах?

\section{МЕТОДЫ ЭКСТРАКЦИИ СПЕРМЫ}

\section{Исследование эякулята}

На сегодняшний день существует несколько современных методов хирургической экстракции сперматозоидов. Однако первым шагом в исследовании фертильности пациента является анализ эякулята. В подавляющем большинстве случаев у мужчин с немозаичной формой СК определяются малое количество эякулята и азооспермия [4]. По данным исследований, при использовании стандартной процедуры анализа эякулята, рекомендуемой ВО3, возможность обнаружения живых сперматозоидов у пациентов с СК составляет около 6-8\% [19, 20]. В то же время Jiang и соавт. были применены цитологические методы анализа, которые повысили процент обнаружения сперматозоидов или герминативных клеток в материале с 6,6 до 21,2\%, большинство из которых были представлены клетками Сертоли. Зарегистрировано несколько случаев беременности и рождения здоровых детей с использованием сперматозоидов из эякулята для экстракорпорального оплодотворения (ЭКО) [21, 22].

Данные о наличии здоровых сперматозоидов у индивидов подросткового возраста противоречивы. По мнению некоторых авторов, шанс обнаружения здоровых сперматозоидов более высок в подростковом возрасте, когда атрофия семенных канальцев еще не столь выражена. Так, Rives et al. выявили олигоастенотератоспермию только у пациента с мозаичной формой СК, a Rohayem et al. выявили олигоастенотератоспермию у пациента с немозаичной формой СК $[23,24]$. С другой стороны, Aksglaede et al. не обнаружили сперматозоиды в эякуляте у индивидов моложе 20 лет [4].

Рядом авторов были предприняты попытки определения предикторных факторов при выявлении здоровых сперматозоидов у пациентов молодого возраста. По данным работы Hagenas et al., в которой исследовалась вероятность содержания зрелых сперматозоидов в эякуляте у пациентов в возрасте 12-18 лет для замораживания перед потенциально гонадотоксичным лечением, единственным предикторным фактором явился объем тестикул более 6 мл. Других предикторов (возраст, уровень тестостерона), способных прогнозировать вероятность положительного исхода исследования эякулята, найдено не было [25]. В противоположность этому, Jiang et al. выявили наличие корреляционной связи между вероятностью получения сперматозоидов в эякуляте и такими факторами, как возраст пациента (чем моложе пациент, тем более высока вероятность обнаружения живых сперматозоидов), уровень Т крови (высокий уровень как положительный фактор) и значение соотношения Т/ЛГ (более высокое значение как положительный фактор) [20]. Важно учитывать, что в связи с физиологическими и психологическими особенностями далеко не все пациенты подросткового возраста готовы обсуждать и планировать вопросы фертильности и будущего потомства, что подтверждают исследования Nahata et al. и Rohayem et al., где около 50\% опрошенных в возрасте от 12 до 25 лет отказались участвовать в исследовании $[24,26]$.

\section{Хирургические методы}

В связи с отсутствием в эякуляте живых сперматозоидов, при их наличии в единичном количестве в просвете канальцев, были разработаны инструментальные методы экстракции зрелых герминативных клеток из тестикулярной ткани. К ним относятся тонкоигольная биопсия, чрескожная биопсия яичка, экстракция сперматозоидов из яичка (testicular sperm extraction, TESE) и микрохирургическая экстракция сперматозоидов яичка (microdissection testicular sperm extraction, mTESE). На сегодняшний день первые два метода практически утратили свою значимость ввиду большей эффективности TESE и $\mathrm{MTESE}$ [27]. 
Техника TESE заключается в надрезании оболочки яичка в одной или нескольких локализациях и экстракции тестикулярной ткани в разном объеме для получения достаточного количества сперматозоидов в биоптате для дальнейшего оплодотворения яйцеклетки. Однако многочисленные надрезы оболочки и довольно обширная экстракция ткани яичка могут привести к внутреннему кровоизлиянию, рубцеванию, необратимому нарушению васкуляризации и дальнейшей атрофии и так частично гиалинизированной ткани органа, что еще более негативно сказывается на гормональном статусе пациента и сперматогенезе [28].

В 1999 г. впервые был применен метод mTESE [29] С возможностью микроскопического распознавания островков сперматогенеза до экстракции ткани. Это значительно повышает вероятность положительного результата и способствует более сохранному состоянию близлежащих тканей, в том числе кровеносных сосудов.

Преимущество $\mathrm{MTESE}$ в том, что процедура не сопровождается большим количеством побочных эффектов [30]. В первой работе, описанной Dr. Peter N. Schlegel, частота положительных результатов экстракции спермы повысилась с 45 до 63\% [29]. Кроме того, при mTESE в биоптате весом в среднем в 9,4 мг удавалось обнаружить 160 тыс. сперматозоидов, в то время как при TESE эта цифра составила 64 тыс. сперматозоидов в 720 мг биоптата (масса биоптата больше в 80 раз). Показательным является и то, что в 6 случаях, в которых применение TESE оказалось неэффективным, был достигнут положительный результат с помощью $\mathrm{mTESE}$.

\section{Эффективность хирургических методов}

Согласно информации из зарубежной литературы, общая вероятность получения здоровых сперматозоидов методом TESE у пациентов с СК составляет от 16\% [31] до 56\% [32], по данным разных авторов. При использовании метода mTESE она возрастает от 20-28\% [33, 34] до 70\% [35]. Коллективом Schiff et al. были предприняты повторные процедуры $\mathrm{mTESE}$, что повысило общий результат эффективности экстракции до 72\% [36].

Имеются данные о применении вышеперечисленных методов у пациентов подросткового возраста, что впервые было описано коллективом Damani et al. в 2001 г. у 15-летнего подростка с положительным эффектом и возможностью дальнейшей криопрезервации [37]. Вместе с тем коллектив Wikström et al., наблюдавший за 14 пациентами с немозаичным вариантом СК в возрасте 10-14 лет, выражает сомнение в целесообразности биопсии и экстракции тестикулярной ткани у подростков, поскольку уже в раннем подростковом возрасте в биоптате отсутствовали клетки на стадии мейоза, но определялись клетки, остановившиеся в развитии на стадии сперматогоний [18].

B группе пациентов Mehta et al. (возраст 14-22 года) результаты $\mathrm{MTESE}$ были выраженно положительными у 7 из 10 подростков были выделены сперматозоиды и заморожены [35]. При этом средний объем тестикул составлял 3,9 мл, всем пациентам была проведена предоперационная гормональная терапия препаратами Т в течение от 1 до 5 лет. Остальные работы завершились менее высокими результатами. В исследовании Rohayem et al. в возрастной группе от 15 до 19 лет доля случаев с положительным исходом mTESE составила 45\%, в то время как в группе 20-45 лет данное значение составило $31 \%$. Наиболее низкие результаты определялись для групп 13-14 лет (10\%) и 45 лет и старше (отрицательные результаты). B работе Nahata et al. у 5 из 10 пациентов (50\%) были обнаружены сперматозоиды в биоптате в результате MTESE [26]. Наименее оптимистичные результаты были получены в 2018 г. исследователями из Бельгии (применялись как TESE, так и MTESE). В возрастной группе 12-16 лет из пяти подростков лишь у одного в биоптате были обнаружены сперматозоиды. Это значительно ниже показателей более взрослой группы пациентов (18-41 год), где сперматозоиды определялись в 48,1\% биоптатов [14]. Оставшиеся биоптаты были исследованы на наличие герминативных клеток. В данном случае клетки чаще определялись именно у подростков, нежели у взрослой группы - 31,5\% и 21\% соответственно. В четырех биоптатах было выявлено малое количество герминативных клеток, что на сегодняшний день не позволяет с уверенностью применять хирургические методы экстракции герминативных клеток у столь юных пациентов.

При применении TESE коллектив Plotton et al. разделил пациентов на две возрастные группы. В группе молодых пациентов от 15 до 22 лет доля положительных исходов манипуляции составила 52\% (у 13 из 25 человек были получены сперматозоиды). И лишь в исследовании Rives et al. изучение биоптата тестикулярной ткани пяти подростков методом TESE выявило наличие сперматозоидов только у одного пациента [23].

Выраженно консервативной позиции придерживается Robert Oates, утверждая, что эуплоидные сперматозоиды, скорее всего, созревают из островков сохранных сперматогониев [38, 39], и экстракция этих островков может сказаться более отрицательно, чем применение выжидательной тактики до взрослого возраста пациента.

Факторы, влияющие на исход применения

хирургических методов

Практически во всех исследованиях предпринимались попытки определения предикторных факторов, позволяющих прогнозировать исход применения как TESE, так и $\mathrm{MTESE}$. Вместе с тем выводы исследователей о наличии предикторных факторов очень противоречивы, а во многих случаях выявить наличие значимой корреляционной связи вовсе не представлялось возможным. Наиболее частым положительным критерием явился возраст пациента до 35 лет [15, 40,41] или даже до 30 лет $[24,33,34]$. Известно, что гиалинизация ткани яичка имеет прогрессирующий характер. Следовательно, было логично ожидать более благоприятный исход операций в молодом возрасте [42, 43]. Кроме того, рядом коллективов уровень Т крови, определяемый до оперативного лечения более 250 нг/дл, в том числе после заместительной гормональной терапии [33, 40,44], также был отмечен как положительный фактор.

Положительным фактором был и гормональный статус, косвенно свидетельствующий об относительной сохранности функции клеток Лейдига, а именно: секреция лг менее 17,5 Ед/л и Т более 7,5 нмоль/л [24, 34, 45], высокий ответ на функциональную пробу с ХГЧ - в среднем 160 нг/дл [44], пороговый объем тестикул в среднем 7,8 мл $[44,46]$ и приближенное к норме соотношение 
концентраций тестостерона и эстрадиола (Т:Э2) [40]. В то же время в исследованиях Nahata et al. и Vicdan et al. не было выявлено ни одного предикторного фактора, а коллективы Corona et al. и Arshad et al. - авторы метаанализов по эффективности вспомогательных репродуктивных технологий у мужчин с СК - утверждают, что ни один из вышеприведенных критериев нельзя считать достоверным $[26,42,43,47,48]$. По данным Seo et al., мозаичность также является положительным прогностическим фактором [31].

К факторам, снижающим вероятность положительного результата операции, относятся дефицит тестостерона $[19,49]$ и низкий уровень андроген-связывающего белка [50]. В исследовании Ozer et al. уровень ФСГ показал отрицательную корреляционную связь [34]. Другие критерии, такие как уровень ингБ, АМГ, не имели статистической значимости ни в одном из исследований.

Тестостерон до экстракции: лечить или не лечить?

Отдельным важным вопросом является влияние терапии Т на исход TESE и mTESE. Несколько авторов указывают на недостаточную доказательную базу для подтверждения или опровержения вреда терапии Т до применения TESE или $\mathrm{mTESE}[47,51]$. Лишь в одном исследовании говорится о возможном отрицательном влиянии терапии Т на исход операции [36]. С тех пор неоднократно продемонстрировано отсутствие существенного влияния предшествующей терапии Т или ингибиторами ароматазы (с предварительной отменой за 6 мес до операции или без прерывания лечения) на результат вмешательства $[32,35]$. Кроме того, ряд авторов обращают внимание на другие, не менее важные эффекты заместительной гормональной терапии: улучшение состава тела, снижение уровня триглицеридов крови, повышение минеральной плотности кости, улучшение концентрации внимания и других когнитивных функций $[52,53]$. Из рисков наиболее настораживают вероятность тромбоэмболии и ускоренное созревание скелета у подростков [54]. Таким образом, терапию андрогенами следует инициировать вне зависимости от дальнейших планов по поводу фертильности [51].

\section{ВСПОМОГАТЕЛЬНЫЕ РЕПРОДУКТИВНЫЕ ТЕХНОЛОГИИ}

Появление вспомогательных репродуктивных технологий в сочетании с современными методами получения сперматозоидов сделало возможным биологическое отцовство для пациентов с СК. Впервые комбинация TESE и интрацитоплазматической инъекции сперматозоида (intracytoplasmic sperm injection, ICSI, метод ИКСИ) у пациентов с СК была применена Palermo et al. в 1998 г. с последующим рождением здорового мальчика у одной из пар и здоровой разнополой двойни - у другой из супружеских пар [55]. К 2017 г., по данным Corona et al., насчитывалось уже более 200 здоровых детей, рожденных благодаря MTESE (или TESE) и ICSI [47], а на сегодняшний день в литературе зафиксировано уже более 300 здоровых родов от отцов с СК $[43,56]$. В большинстве исследований определяется высокая доля оплодотворенных яйцеклеток, в среднем составляющая 51,5\%, а максимально указанная Friedler et al. - 66\% [57]. Ca- мая малая степень оплодотворения указана в работе Sabbaghian et al. - 28\% [33], которая все же была значимо более высокой у группы пациентов с СК в сравнении с контрольной группой. Прямо противоположны этому результату данные Bakircioglu et al., в работе которых продемонстрирована более низкая степень оплодотворения в сравнении с контрольной группой мужчин с иными формами необструктивной азооспермии (57\% для СК и 65\% для контрольной группы) [41]. Недавно опубликованы данные китайских исследователей Zhang et al. на основе терапии 284 пациентов с CK. При доле удачных $\mathrm{mTESE}$ в 44\%, оплодотворение прошло успешно в $54 \%$ случаев и в 50,4\% случаев закончилось родами здоровых детей - всего 63 ребенка [56].

В ряде работ проведено сравнение использования свежей или замороженной спермы, однако статистически значимых отличий в эффективности лечения выявлено не было. Например, Friedler et al. сравнивали степень оплодотворения яйцеклеток (66\% и 58\% для свежей и замороженной спермы соответственно), степень дробления (98\% и 90\%), а также количество удачных имплантаций (33,3\% и 21,4\%) - ни в одном из параметров статистически значимых отличий найдено не было [57]. Схожие данные получены китайским коллективом Chen et al. - ученые не отмечали статистически значимой разницы между применением размороженных (38 пациентов) или свежих (155 пациентов) сперматозоидов [46]. Многие работы с использованием замороженной спермы подтверждают, что качество сперматозоидов позволяет добиться здоровой беременности и здорового потомства [21, 48, 58-60].

\section{Здоровье потомства}

При обеспечении биологического отцовства у пациентов ССК немаловажное значение имеет здоровье детей. Поскольку СК по этиологии относится к заболеваниям с увеличенным числом хромосом, вполне вероятно наличие подобных нарушений и потомства. Действительно, в нескольких работах было продемонстрировано наличие гамет с аномальным набором как половых хромосом, так и аутосом. Еще в 1999 г. Foresta et al. показали присутствие сперматогониев с хромосомным набором 47,XXY в 100\% исследуемых клетках, а также наличие доли вторичных сперматоцитов, сперматидов и сперматозоидов с хромосомным набором 24,XX и 24,XY до 30,0\% [3]. В работе Estop et al. 50\% всех исследуемых сперматозоидов (26 клеток) имели аномальную хромосомную структуру с различными вариантами набора половых хромосом и 18-й аутосомы [61]. Более оптимистичные результаты продемонстрировал коллектив Levron et al., проанализировав 112 сперматозоидов, среди которых лишь 6,7\% оказались с хромосомными нарушениями [62]. К вариантам с нарушениями относились гаметы 24XX, 24XY, 24YY, 2200 и две гаметы с отсутствующей 18-й аутосомой. Схожие данные продемонстрированы Yamamoto et al., выявившими значительно большее количество хромосомных аномалий в группе с СК, нежели в контрольной группе мужчин с необструктивной азооспермией иного генеза [50]. Наиболее ободряющие результаты получены коллективом Sciurano et al. в 2009 г. - все 92 исследуемых сперматоцита были эуплоидными, а клетки Сертоли имели аномальный набор хромосом 47XXY [38]. Авторы 
рассуждают, что описанные эуплоидные клетки, скорее всего, происходят от тех сперматогониев, которые случайным образом теряют лишнюю Х-хромосому в течение созревания.

В нескольких случаях сообщалось о наличии эмбрионов с набором хромосом 47XXY [63] и в двух случаях о прерывании беременности в связи с аномальным набором хромосом [57, 60]. В исследовании Vicdan et al. у 12 детей из 21 родившихся после TESE и ИКСИ проводилось кариотипирование - нарушений набора хромосом выявлено не было. В данном исследовании описывались также 3 случая смерти новорожденных по причине недоношенности и малого веса, 1 случай смерти в связи с рядом аномалий развития [48]. В остальных случаях задокументировано рождение здоровых детей.

\section{ЗАКЛЮЧЕНИЕ}

Обобщая вышеизложенное, можно заключить, что современные методы экстракции сперматозоидов TESE и $\mathrm{MTESE}$ - являются эффективными технологиями для предоставления возможности отцовства мужчинам с СК. В среднем возможность получения жизнеспособных сперматозоидов составляет 50-55\%, а оптимальным периодом проведения манипуляций можно считать возраст от 18 до 30 лет вне зависимости от готовности к отцовству в данный период жизни (возможна криопрезервация спермы). Проведение указанных вмешательств пациентам младше 16 лет на сегодняшний день эксперты не рекомендуют ввиду отсутствия доказательств эффективности такой тактики. Помимо возраста, других предикторных факторов выявить пока не удалось.

По данным ряда исследований, терапия препаратами тестостерона не имеет отрицательного влияния на исход TESE или mTESE. Это говорит о том, что ее можно назначать в соответствии с клиническими рекомендациями.

В нескольких исследованиях были показаны клетки с аномальным набором хромосом после оплодотворения. Вместе с тем во всех исследованиях, представленных в данном обзоре, задокументировано рождение здоровых детей, что свидетельствует о, скорее, низком риске передачи хромосомных аномалий потомству. И все же медико-генетическое консультирование с разъяснением рисков, а также предимплантационная или внутриутробная диагностика являются обязательной частью вспомогательных репродуктивных технологий у мужчин с CK [21].

\section{ДОПОЛНИТЕЛЬНАЯ ИНФОРМАЦИЯ}

Источник финансирования. Работа выполнена при поддержке гранта Министерства науки и высшего образования Российской Федерации (соглашение № 075-15-2020-899).

Конфликт интересов. Авторы декларируют отсутствие явных и потенциальных конфликтов интересов, связанных с публикацией настоящей статьи.

Участие авторов. Все авторы одобрили финальную версию статьи перед публикацией, выразили согласие нести ответственность за все аспекты работы, подразумевающую надлежащее изучение и решение вопросов, связанных с точностью или добросовестностью любой части работы.

\section{СПИСОК ЛИТЕРАТУРЫ | REFERENCES}

1. Сафина Н.Ю, Яманди Т.А., Черных В.Б., и др. Генетические факторы мужского бесплодия, их сочетания и спермиологическая характеристика мужчин с нарушением фертильности. Андрология и генитальная хирургия. - 2018. - Т. 19. №2. - C. 40-51. [Safina NYu, Yamandi TA, Chernykh VB, et al. Genetic factors of male infertility, their combinations and the spermatological characteristics of men with fertility failures. Andrology and Genital Surgery. 2018;19(2):40-51. (In Russ.)]. doi: https://doi.org/10.17650/2070-9781-2018-19-2-40-51

2. Yoshida A, Miura K, Shirai M. Cytogenetic Survey of 1,007 Infertile Males. Urol Int. 1997;58(3):166-176. doi: https://doi.org/10.1159/000282975.

3. Foresta $C$, et al. Analysis of meiosis in intratesticular germ cells from subjects affected by classic Klinefelter's syndrome. J Clin Endocrinol Metab. 1999;84(10):3807-3810. doi: https://doi.org/10.1210/jcem.84.10.6029

4. Aksglaede L, et al. 47,XXY Klinefelter syndrome: clinical characteristics and age-specific recommendations for medical management. Am J Med Genet C Semin Med Genet. 2013;163c(1):55-63. doi: https://doi.org/10.1002/ajmg.c.31349

5. Groth KA, et al. Clinical review: Klinefelter syndrome a clinical update. J Clin Endocrinol Metab. 2013;98(1):20-30. doi: https://doi.org/10.1210/jc.2012-2382

6. Bojesen A, Juul S, Gravholt CH. Prenatal and postnatal prevalence of Klinefelter syndrome: a national registry study. J Clin Endocrinol Metab. 2003;88(2):622-626. doi: https://doi.org/10.1210/jc.2002-021491

7. Herlihy AS, McLachlan RI. Screening for Klinefelter syndrome. Curr Opin Endocrinol Diabetes Obes. 2015;22(3):224-229. doi: https://doi.org/10.1097/med.0000000000000154

8. Klinefelter $\mathrm{H}$, et al. Syndrome Characterized by Gynecomastia, Aspermatogenesis without A-Leydigism, and Increased Excretion of Follicle-Stimulating Hormone. The Journal of Clinical Endocrinology \& Metabolism. 1942;2(11):615-627. doi: https://doi.org/10.1210/jcem-2-11-615
9. Gravholt $\mathrm{CH}$, et al. Body composition, metabolic syndrome and type 2 diabetes in Klinefelter syndrome. Acta Paediatr. 2011;100(6):871-877. doi: https://doi.org/10.1111/j.1651-2227.2011.02233.x

10. Ross JL, et al. Cognitive and motor development during childhood in boys with Klinefelter syndrome. Am J Med Genet $A$. 2008;146a(6):708-719. doi: https://doi.org/10.1002/ajmg.a.32232

11. Bojesen A, Gravholt CH. Morbidity and mortality in Klinefelter syndrome (47,XXY). Acta Paediatr. 2011;100(6):807-813. doi: https://doi.org/10.1111/j.1651-2227.2011.02274.x

12. Coerdt W, et al. Quantitative histology of human fetal testes in chromosomal disease. Pediatr Pathol. 1985;3(2-4):245-259.

13. Winge SB, et al. Transcriptome profiling of fetal Klinefelter testis tissue reveals a possible involvement of long non-coding RNAs in gonocyte maturation. Hum Mol Genet. 2018;27(3):430-439. doi: https://doi.org/10.1093/hmg/ddx411

14. Van Saen D, et al. When does germ cell loss and fibrosis occur in patients with Klinefelter syndrome? Hum Reprod. 2018;33(6):1009-1022. doi: https://doi.org/10.1093/humrep/dey094

15. Shiraishi K, Matsuyama H. Klinefelter syndrome: From pediatrics to geriatrics. Reprod Med Biol. 2019;18(2):140-150. doi: https://doi.org/10.1002/rmb2.12261

16. Lahlou $\mathrm{N}$, et al. Inhibin B and anti-Mullerian hormone, but not testosterone levels, are normal in infants with nonmosaic Klinefelter syndrome. J Clin Endocrinol Metab. 2004;89(4):1864-1868. doi: https://doi.org/10.1210/jc.2003-031624

17. Aksglaede $L$, et al. High normal testosterone levels in infants with non-mosaic Klinefelter's syndrome. Eur J Endocrinol. 2007;157(3):345-350. doi: https://doi.org/10.1530/eje-07-0310

18. Wikstrom AM, et al. Klinefelter syndrome in adolescence: onset of puberty is associated with accelerated germ cell depletion. J Clin Endocrinol Metab. 2004;89(5):2263-2270. doi: https://doi.org/10.1210/jc.2003-031725 
19. Selice $R$, et al. Spermatogenesis in Klinefelter syndrome. J Endocrinol Invest. 2010;33(11):789-793. doi: https://doi.org/10.3275/6935

20. Jiang YT, et al. Sperm retrieval from patients with nonmosaic Klinefelter's syndrome by semen cytology examination. Genet Mol Res. 2014;13(1):1848-1854. doi: https://doi.org/10.4238/2014.March.17.12

21. Staessen C, et al. PGD in $47, X X Y$ Klinefelter's syndrome patients. Hum Reprod Update. 2003;9(4):319-330.

22. Tachdjian G. Reproductive genetic counselling in non-mosaic 47,XXY patients: implications for preimplantation or prenatal diagnosis: Case report and review. Hum Reprod. 2003;18(2):271-275. doi: https://doi.org/10.1093/humrep/deg070.

23. Rives $\mathrm{N}$, et al. The feasibility of fertility preservation in adolescents with Klinefelter syndrome. Hum Reprod. 2013;28(6):1468-1479. doi: https://doi.org/10.1093/humrep/det084

24. Rohayem J, et al. Age and markers of Leydig cell function, but not of Sertoli cell function predict the success of sperm retrieval in adolescents and adults with Klinefelter's syndrome. Andrology. 2015;3(5):868-875. doi: https://doi.org/10.1111/andr.12067

25. Hagenas I, et al. Clinical and biochemical correlates of successful semen collection for cryopreservation from 12-18-year-old patients: a single-center study of 86 adolescents. Hum Reprod. 2010;25(8):2031-2038. doi: https://doi.org/10.1093/humrep/deq147

26. Nahata $L$, et al. Sperm Retrieval in Adolescents and Young Adults with Klinefelter Syndrome: A Prospective, Pilot Study. J Pediatr. 2016;170:260-265.e261-262. doi: https://doi.org/10.1016/j.jpeds.2015.12.028

27. Dabaja AA, Schlegel PN. Microdissection testicular sperm extraction: an update. Asian J Androl. 2013;15(1):35-39. doi: https://doi.org/10.1038/aja.2012.141

28. Ramasamy R, Yagan N, and Schlegel PN. Structural and functional changes to the testis after conventional versus microdissection testicular sperm extraction. Urology. 2005;65(6):1190-1194. doi: https://doi.org/10.1016/j.urology.2004.12.059

29. Schlegel PN. Testicular sperm extraction: microdissection improves sperm yield with minimal tissue excision. Hum Reprod. 1999;14(1):131-135. doi: https://doi.org/10.1093/humrep/14.1.131.

30. Amer M, Ateyah A, Hany R, Zohdy W. Prospective comparative study between microsurgical and conventional testicular sperm extraction in non-obstructive azoospermia: follow-up by serial ultrasound examinations. Hum Reprod. 2000;15(3):653-656. doi: https://doi.org/10.1093/humrep/15.3.653.

31. Seo JT, Park YS, Lee JS. Successful testicular sperm extraction in Korean Klinefelter syndrome. Urology. 2004;64(6):1208-1211. doi: https://doi.org/10.1016/j.urology.2004.07.013

32. Plotton I, et al. Preliminary Results of a Prospective Study of Testicular Sperm Extraction in Young Versus Adult Patients With Nonmosaic 47,XXY Klinefelter Syndrome. J Clin Endocrinol Metab. 2015;100(3):961-967. doi: https://doi.org/10.1210/jc.2014-3083

33. Sabbaghian $\mathrm{M}$, et al. Comparison of sperm retrieval and intracytoplasmic sperm injection outcome in patients with and without Klinefelter syndrome. Urology. 2014;83(1):107-110. doi: https://doi.org/10.1016/j.urology.2013.09.021

34. Ozer C, Caglar Aytac P, Goren MR, et al. Sperm retrieval by microdissection testicular sperm extraction and intracytoplasmic sperm injection outcomes in nonobstructive azoospermic patients with Klinefelter syndrome. Andrologia. 2018;50(4):e12983. doi: https://doi.org/10.1111/and.12983

35. Mehta A, et al. Successful testicular sperm retrieval in adolescents with Klinefelter syndrome treated with at least 1 year of topical testosterone and aromatase inhibitor. Fertil Steril. 2013;100(4):970-974. doi: https://doi.org/10.1016/j.fertnstert.2013.06.010

36. Schiff JD, et al. Success of testicular sperm extraction [corrected] and intracytoplasmic sperm injection in men with Klinefelter syndrome. J Clin Endocrinol Metab. 2005;90(11):6263-6267. doi: https://doi.org/10.1210/jc.2004-2322

37. Damani MN, Mittal R, and Oates RD. Testicular tissue extraction in a young male with 47,XXY Klinefelter's syndrome: potential strategy for preservation of fertility. Fertil Steril. 2001;76(5):1054-1056. doi: https://doi.org/10.1016/s0015-0282(01)02837-0

38. Sciurano RB, et al. Focal spermatogenesis originates in euploid germ cells in classical Klinefelter patients. Hum Reprod. 2009;24(9):2353-2360. doi: https://doi.org/10.1093/humrep/dep180

39. Oates R. Adolescent Klinefelter syndrome: is there an advantage to testis tissue harvesting or not? F1000Res. 2016;5(2):1595. doi: https://doi.org/10.12688/f1000research.8395.1
40. Ramasamy $R$, et al. Successful fertility treatment for Klinefelter's syndrome. J Urol. 2009;182(3):1108-1113. doi: https://doi.org/10.1016/j.juro.2009.05.019

41. Bakircioglu ME, et al. Klinefelter syndrome: does it confer a bad prognosis in treatment of nonobstructive azoospermia? Fertil Steril. 2011;95(5):1696-1699. doi: https://doi.org/10.1016/jffertnstert.2011.01.005

42. Arshad MA, Majzoub A, Esteves SC. Predictors of surgical sperm retrieval in non-obstructive azoospermia: summary of current literature. Int Urol Nephrol. 2020;52(11):2015-2038. doi: https://doi.org/10.1007/s11255-020-02529-4

43. Chen W, Bai MZ, Yang Y, et al. ART strategies in Klinefelter syndrome. J Assist Reprod Genet. 2020;37(9):2053-2079. doi: https://doi.org/10.1007/s10815-020-01818-2

44. Madgar I. Prognostic value of the clinical and laboratory evaluation in patients with nonmosaic Klinefelter syndrome who are receiving assisted reproductive therapy. Fertil Steril. 2002;77(6):1167-1169. doi: https://doi.org/10.1016/S0015-0282(02)03092-3.

45. Витязева И.И, Боголюбов С.В., Мун Т.В., и др. Собственные результаты лечения пациентов с немозаичной формой синдрома Клайнфельтера в программе ЭКО-ИКСИ-микро-ТЕSЕ // Проблемь репродукции. - 2017. - Т. 23. - №5. - C. 65-72. [Vityazeva II, Bogolyubov SV, Mun TV, et al. The results of treatment of patients with non-mosaic form of Klinefelter syndrome in the IVF/ICSI/microTESE program. Problemy reproduktsii. 2017;23(5):65-72. (In Russ.)]. doi: https://doi.org/10.17116/repro201723565-72

46. Chen $\mathrm{X}$, et al. Comparison and outcomes of nonobstructive azoospermia patients with different etiology undergoing MicroTESE and ICSI treatments. Trans/ Androl Urol. 2019;8(4):366-373. doi: https://doi.org/10.21037/tau.2019.04.08

47. Corona G, et al. Sperm recovery and ICSI outcomes in Klinefelter syndrome: a systematic review and metaanalysis. Hum Reprod Update. 2017;23(3):265-275. doi: https://doi.org/10.1093/humupd/dm×008

48. Vicdan $\mathrm{K}$, et al. Outcome of intracytoplasmic sperm injection using fresh and cryopreserved-thawed testicular spermatozoa in 83 azoospermic men with Klinefelter syndrome. J Obstet Gynaecol Res. 2016;42(11):1558-1566. doi: https://doi.org/10.1111/jog.13090

49. Витязева И.И, Боголюбов С.В., Брагина Е.Е., и др. Возможность получения сперматозоидов у мужчин с немозаичной формой синдрома Клайнфельтера в программах экстракорпорального оплодотворения. Обзор литературы и описание случая // Андрология и генитальная хирургия. - 2014. - Т. 15. — № 3. C. 16-25. [Vityazeva II, Bogolyubov SV, Bragina YY., et al. Microdissection testicular sperm extraction in patients with non-mosaic klinefelter syndrome in programme in vitro fertilization: literature review and cases report. Andrologiya igenital'naya khirurgiya. 2014;15(3):16-25. (In Russ.)]. doi: https://doi.org/10.17650/2070-9781-2014-3-16-25

50. Yamamoto Y. Morphometric and cytogenetic characteristics of testicular germ cells and Sertoli cell secretory function in men with non-mosaic Klinefelter's syndrome. Hum Reprod. 2002;17(4):886-896. doi: https://doi.org/10.1093/humrep/17.4.886.

51. Franik S, et al. Klinefelter syndrome and fertility: sperm preservation should not be offered to children with Klinefelter syndrome. Hum Reprod. 2016;31(9):1952-1959. doi: https://doi.org/10.1093/humrep/dew179

52. Davis SM, et al. Effects of Oxandrolone on Cardiometabolic Health in Boys With Klinefelter Syndrome: A Randomized Controlled Trial. J Clin Endocrinol Metab. 2017;102(1):176-184 doi: https://doi.org/10.1210/jc.2016-2904

53. Samango-Sprouse $C$, et al. Positive effects of early androgen therapy on the behavioral phenotype of boys with 47,XXY. Am J Med Genet C Semin Med Genet. 2015;169(2):150-157. doi: https://doi.org/10.1002/ajmg.c.31437

54. Martinez C, et al. Testosterone treatment and risk of venous thromboembolism: population based case-control study. Bmj. 2016;355:5968. doi: https://doi.org/10.1136/bmj.i5968

55. Palermo GD, et al. Births after intracytoplasmic injection of sperm obtained by testicular extraction from men with nonmosaic Klinefelter's syndrome. N Eng/ J Med. 1998;338(9):588-590. doi: https://doi.org/10.1056/nejm199802263380905

56. Zhang H-L, Zhao L-M, Mao J-M, et al. Sperm retrieval rates and clinical outcomes for patients with different causes of azoospermia who undergo microdissection testicular sperm extractionintracytoplasmic sperm injection. Asian J Androl. 2020;9(7):719. doi: https://doi.org/10.4103/aja.aja_12_20 
57. Friedler S, Raziel A, Strassburger D, Schachter M, Bern O, Ron-El R. Outcome of ICSI using fresh and cryopreservedthawed testicular spermatozoa in patients with non-mosaic Klinefelter's syndrome. Hum Reprod. 2001;16(12):2616-2620. doi: https://doi.org/10.1093/humrep/16.12.2616.

58. Kyono K, et al. Seven pregnancies and deliveries from nonmosaic Klinefelter syndrome patients using fresh and frozen testicular sperm. J Assist Reprod Genet. 2007;24(1):47-51. doi: https://doi.org/10.1007/s10815-006-9079-4

59. Madureira C, et al. Treatment by testicular sperm extraction and intracytoplasmic sperm injection of 65 azoospermic patients with non-mosaic Klinefelter syndrome with birth of 17 healthy children. Andrology. 2014;2(4):623-631. doi: https://doi.org/10.1111/j.2047-2927.2014.00231.x

60. Ron-EI R. A 47,XXY fetus conceived after ICSI of spermatozoa from a patient with non-mosaic Klinefelter's syndrome:
Case report. Hum Reprod. 2000;15(8):1804-1806. doi: https://doi.org/10.1093/humrep/15.8.1804.

61. Estop AM, Munne S, Cieply KM, et al. Meiotic products of a Klinefelter 47,XXY male as determined by sperm fluorescence in-situ hybridization analysis. Hum Reprod. 1998;13(1):124-127. doi: https://doi.org/10.1093/humrep/13.1.124.

62. Levron J, Aviram-Goldring A, Madgar I, et al. Sperm chromosome analysis and outcome of IVF in patients with non-mosaic Klinefelter's syndrome. Fertil Steril. 2000;74(5):925-929. doi: https://doi.org/10.1016/S0015-0282(00)01556-9.

63. Reubinoff $\mathrm{BE}$, Abeliovich $\mathrm{D}$, Werner $\mathrm{M}$, et al. A birth in nonmosaic Klinefelter's syndrome after testicular fine needle aspiration, intracytoplasmic sperm injection and preimplantation genetic diagnosis. Hum Reprod. 1998;13(7):1887-1892. doi: https://doi.org/10.1093/humrep/13.7.1887.

Рукопись получена: 15.09.2020. Одобрена к публикации: 29.11.2020. Опубликована online: 30.12.2020.

\section{ИНФОРМАЦИЯ ОБ АВТОРАХ [AUTHORS INFO]}

*Воронцова Мария Владимировна, к.м.н. [Maria V. Vorontsova, MD, PhD]; адрес: Россия, 117036, Москва, ул. Дм. Ульянова, д. 11 [address: 11 Dm. Ulyanova street, 117036 Moscow, Russia]; ORCID: https://orcid.org/0000-0002-9124-294X; eLibrary SPIN: 4168-6851; e-mail: maria.vorontsova.endo@gmail.com.

Калинченко Наталья Юрьевна, К.M.H. [Natalia Yu. Kalinchenko, MD, PhD]; ORCID: https://orcid.org/0000-0002-2000-7694, eLibrary SPIN: 6727-9653; e-mail: kalinnat@rambler.ru.

\section{ЦИТИРОВАТЬ:}

Воронцова М.В., Калинченко Н.Ю. Синдром Клайнфельтера: обзор литературы по использованию современных методов вспомогательных репродуктивных технологий // Проблемы эндокринологии. - 2020. — Т. 66. — №6. — С. 31-38. doi: https://doi.org/10.14341/probl12666

\section{TO CITE THIS ARTICLE:}

Vorontsova NV, Kalinichenko NY. Klinefelter syndrome: literature review on using modern methods of assisted reproductive technologies. Problems of Endocrinology. 2020;66(6):31-38. doi: https://doi.org/10.14341/probl12666 